\title{
Silencing LDHA inhibits proliferation, induces apoptosis and increases chemosensitivity to temozolomide in glioma cells
}

\author{
HUI DI $^{1}$, XINTING ZHANG ${ }^{2}$, YI GUO $^{3}$, YANFANG SHI ${ }^{1}$, CHUAN FANG $^{1}$, YU YUAN $^{1}$, \\ JIWEI WANG ${ }^{1}, \mathrm{CHAO} \mathrm{SHANG}^{4}$, WENZHE GUO ${ }^{1}$ and $\mathrm{CHUNHUI} \mathrm{LI}^{1}$ \\ ${ }^{1}$ Department of Neurosurgery, Affiliated Hospital of Hebei University; ${ }^{2}$ Department of Neurosurgery, Hebei University, \\ Baoding, Hebei 071000; ${ }^{3}$ Department of Neurosurgery, Beijing Tsinghua Changgung Hospital, Tsinghua University, \\ Beijing 102218; ${ }^{4}$ Department of Neurosurgery, Tianjin Huanhu Hospital, Tianjin 300350, P.R. China
}

Received April 2, 2017; Accepted October 5, 2017

DOI: $10.3892 / 01.2018 .7932$

\begin{abstract}
Glioblastoma multiforme (GBM) is a prevalent and aggressive disease, and the development of a novel therapy to better treat advanced GBM is urgently required. Lactate dehydrogenase A (LDHA), which functions as a key enzyme in transforming pyruvate into lactate, has attracted more attention in recent years due to its critical role in various types of advanced cancer. Previous data derived from the Oncomine database have shown that the expression of LDHA is higher in GBM tissues than that in corresponding normal control tissues. However, the association of LDHA levels with glioma clinical grades and the possible mechanisms of LDHA in GBM progression have not been investigated. The present study showed that there is a significant positive correlation between LDHA expression levels and tumor clinical stages. The knockdown of LDHA inhibited cell growth by inhibiting cell cycle progression and inducing apoptosis in glioma cell lines. Upon investigating the molecular mechanism, LDHA knockdown via siRNA treatment was associated with decreased cyclin D1 expression, increased cleavage of PARP, and altered B-cell lymphoma 2 and B-cell lymphoma 2-associated protein $\mathrm{X}$ expression. In addition, LDHA knockdown led to the marked downregulation of matrix metalloproteinase (MMP)-2, MMP-9, VE-Cadherin and vascular endothelial growth factor expression levels. Furthermore, knock down of LDHA enhanced the chemosensitivity of glioma cells to temozolomide (TMZ), a second-generation alkylating agent with activity against recurrent high-grade glioma. These findings
\end{abstract}

Correspondence to: Dr Chunhui Li, Department of Neurosurgery, Affiliated Hospital of Hebei University, 212 Yu Hua Earth Road, Baoding, Hebei 071000, P.R. China

E-mail: lichunhui312@126.com

Abbreviations: LDHA, lactate dehydrogenase A; GBM, glioblastoma; TMZ, Temozolomide; PCR, polymerase chain reaction

Key words: lactate dehydrogenase A, glioblastoma multiforme, Temozolomide, proliferation, apoptosis support LDHA as a novel target for developing effective therapeutic strategies to treat GBM.

\section{Introduction}

Glioblastoma multiforme (GBM) is the most common and malignant brain tumor and has a median survival rate of less than two years $(1,2)$. Current therapies, including surgery, radiotherapy and chemotherapy, are generally ineffective at controlling the progression and development of GBM, and recurrence is practically inevitable (3). Therefore, it is important to understand the molecular mechanisms that are responsible for the abnormal features of GBM and identify new therapeutic targets.

The Warburg effect, also known as aerobic glycolysis, is an emerging hallmark of tumor cell metabolism (4-6). Lactate dehydrogenase A (LDHA) is a key enzyme in aerobic glycolysis that increases glucose uptake and lactate production in tumor cells; LDHA has attracted increasing attention in recent years due to its critical role in tumor progression (7-9). In breast cancer, LDHA knockdown suppresses tumor progression by inducing mitochondrial pathway apoptosis (10). In oesophageal squamous cell carcinoma (ESCC), knocking down LDHA inhibited cell growth and migration in vitro, as well as tumorigenesis in vivo (11). In hepatocellular carcinoma (HCC), LDHA knockdown resulted in a significant reduction in metastatic potential in a xenograft mouse model (12). In pancreatic cancer, when LDHA was overexpressed, tumor growth was increased (13). In renal cell carcinoma, knocking down LDHA resulted in potential anticancer effects (14). Although the abnormal expression of LDHA has been reported in several cancers (10-15), little is known about the expression pattern of LDHA and its underlying roles in GBM. Previous reports using data from the Oncomine database showed that the mRNA levels of LDHA were up-regulated in GBM compared to matched tissues (16); these finding prompted us to examine the underlying role of LDHA in glioma malignancy.

In the present study, we investigated the relationship between LDHA expression levels and GBM malignancy; we also explored the effects of LDHA knockdown on proliferation and apoptosis and its underlying mechanisms, and we further determined whether the inhibition of LDHA could be used to 
increase the chemosensitivity of glioma cells to temozolomide (TMZ).

\section{Materials and methods}

Patient glioma samples. Human glioma samples were obtained from patients of the Department of Neurosurgery, Affiliated Hospital of Hebei University, and the study was approved by the Ethics Committee of the Affiliated Hospital of Hebei University. The human glioma tissue samples used in this study were from 10 patients with grade II GBM, 7 patients with grade III GBM and 9 patients with grade IV GBM; these patients were derived according to the World Health Organization (WHO) classification.

Cells and reagents. The human glioma cell lines U87 and U251 were obtained from Peking Union Medical College Cell Library (Beijing, China) and were maintained in Dulbecco's modified Eagle's medium (DMEM) medium supplemented with $10 \%$ heat inactivated foetal bovine serum (FBS), 100 units $/ \mathrm{ml}$ penicillin, and $100 \mathrm{mg} / \mathrm{ml}$ streptomycin at $37^{\circ} \mathrm{C}$ in a humidified atmosphere containing $5 \% \mathrm{CO}_{2}$. All media and sera were purchased from Gibco (Thermo Fisher Scientific, Inc., Waltham, MA, USA).

Antibodies against LDHA (3582, 1:1,000), Bax (5023, 1:1,000), Bcl-2 (3498, 1:1,000), PARP (9532, 1:1,000), cyclin D1 (2978, 1:1,000), VE-cadherin $(2500,1: 1,000)$ and GAPDH (5174, 1:1,000) were from Cell Signalling Technology, Inc. (Danvers, MA, USA). Antibodies against MMP-2 (sc-53630, 1:500), MMP-9 (sc-21736, 1:500) and VEGF (sc-365578, 1:500) were from Santa Cruz Biotechnology, Inc. (Dallas, TX, USA).

siRNA transfection. The sequence of siRNA targeting LDHA (GenePharma, Shanghai, China) has been described previously: 5'-CGAACTGGGCAGTATAAAC-3'; negative control (NC), 5'-UUCUCCGAACGUGUCACGUTT-3' ${ }^{14}$. U87 and U251 cells were transfected with specific siRNAs using the Lipofectamine 2000 reagent in antibiotic-free Opti-MEM medium (both from Invitrogen; Thermo Fisher Scientific, Inc.) according to the manufacturer's protocol.

Western blotting. U87 and U251 cells were added to radioimmunoprecipitation assay (RIPA) lysis buffer (CW Biotech, Beijing, China) containing a protease inhibitor cocktail (Roche Diagnostics, Basel, Switzerland) and incubated for $30 \mathrm{~min}$ at $4^{\circ} \mathrm{C}$. After sodium dodecyl sulfate polyacrylamide gel electrophoresis (SDS-PAGE), the proteins were transferred onto nitrocellulose membranes and blocked with $5 \%$ milk in TBST buffer (20 mM Tris-HCl, pH 7.4; $137 \mathrm{mM} \mathrm{NaCl}$; and $0.1 \%$ Tween-20) for $1 \mathrm{~h}$ at $37^{\circ} \mathrm{C}$. Then, the cells were incubated overnight at $4^{\circ} \mathrm{C}$ with primary antibodies against the target proteins. The membranes were washed five times and incubated with the appropriate horseradish peroxidase (HRP)-conjugated secondary antibody for $90 \mathrm{~min}$ at room temperature; the membranes were then developed using ECL reagents (EMD Millipore, Billerica, MA, USA).

Quantitative PCR ( $q P C R)$. Total RNA was extracted from cultured U87 and U251 cells using TRIzol reagent (Invitrogen; Thermo Fisher Scientific, Inc.), and cDNA

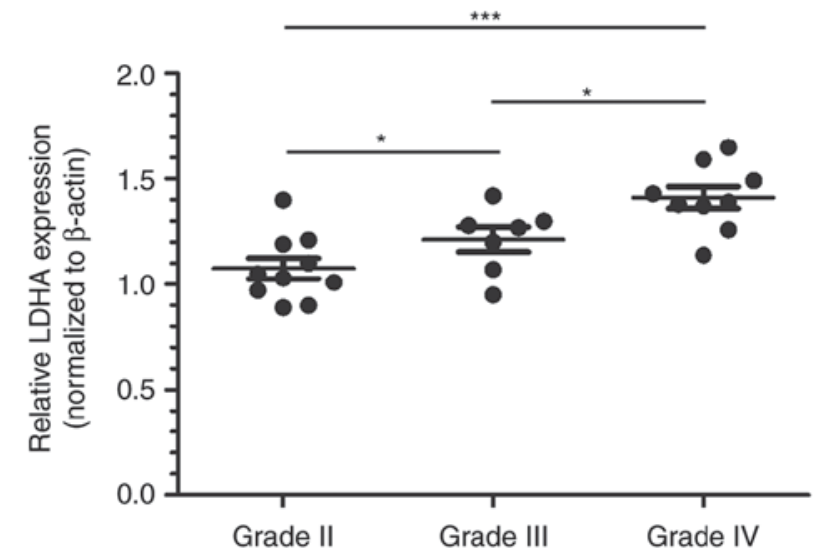

Figure 1. Relative expression levels of LDHA in 3 different grades of glioma samples analysed by qRT-PCR. Grade II (10 glioma samples), grade III (7 glioma samples), and grade IV (9 glioma samples). The data are presented as the mean \pm SD. ${ }^{*} \mathrm{P}<0.05,{ }^{* * *} \mathrm{P}<0.005$. LDHA, lactate dehydrogenase A.

was synthesized using the RevertAid First-Strand cDNA Synthesis kit (Thermo Fisher Scientific, Inc.). qRT-PCR was performed using a MasterCycler system (Eppendorf AG, Hamburg, Germany) and the FastStart Universal SYBR Green Master kit (Roche Diagnostics, Indianapolis, IN, USA). Primer sequences were as follows: LDHA forward, 5'-CTCCTGTGCAAAATGGCAAC-3' and reverse, 5'-CCT AGAGCTCACTAGTCACAG-3'; $\beta$-actin forward, 5'-CGT GACATTAAGGAGAAGCTG-3' and reverse, 5'-CTAGAA GCATTTGCGGTGGAC-3'. The relative expression of LDHA was calculated by using the $\Delta-\mathrm{Ct}$ of the target gene and normalized to the expression of $\beta$-actin.

Cell viability assay. U87 or U251 cells transfected with si-NC or si-LDHA were seeded in 96-well plates at 5,000 cells/well. After the samples were incubated with 3-(4,5-dimethylthiazol-2-yl)-2,5-diphenyl-tetrazolium bromide (MTT) dissolved in $150 \mu \mathrm{l}$ of dimethyl sulfoxide (DMSO) for 4-6 h at $37^{\circ} \mathrm{C}$, the absorbance was measured at $490 \mathrm{~nm}$ using a microplate reader.

Cell cycle analysis. Cell cycle analysis was performed using propidium iodide (PI) stain (BD Biosciences, Franklin Lakes, NJ, USA) according to the manufacturer's instructions. Briefly, U87 and U251 cells $\left(5 \times 10^{5}\right)$ transfected with si-NC or si-LDHA were harvested, fixed in $5 \mathrm{ml}$ of ice-cold $70 \%$ ethanol overnight, and incubated with $50 \mu \mathrm{g} / \mathrm{ml} \mathrm{PI}$ at $4^{\circ} \mathrm{C}$; then, the DNA content was determined using a FACSVerse flow cytometer (BD Biosciences). The percentages of cells in G0/G1, S, and $\mathrm{G} 2 / \mathrm{M}$ phases are presented quantitatively.

Apoptosis assay. Apoptosis assays were performed using an Annexin V-FITC apoptosis detection kit (BD Biosciences) according to the manufacturer's instructions. Briefly, U87 and U251 cells were harvested and stained with Annexin V-FITC and PI in the dark for $15 \mathrm{~min}$ at room temperature. Apoptotic cells were quantitatively analysed using a FACSVerse flow cytometer and FACSuite analysis software (BD Biosciences). The total percentages of Annexin V-positive cells are presented quantitatively. 
A
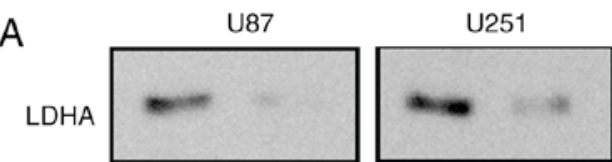

GAPDH

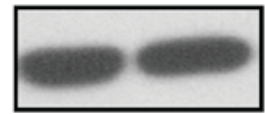

si-NC si-LDHA

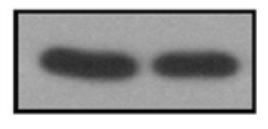

si-NC si-LDHA

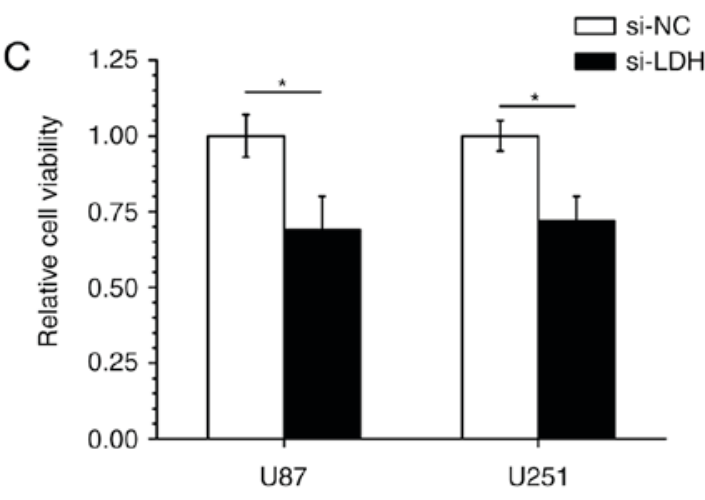

D
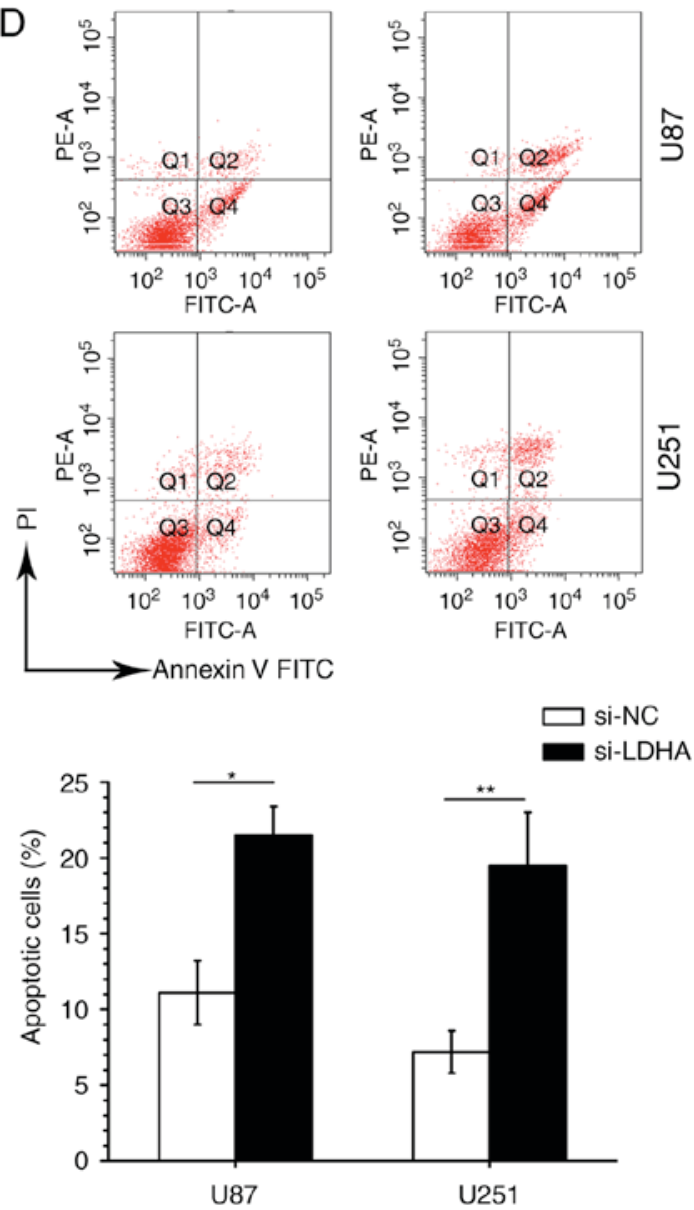
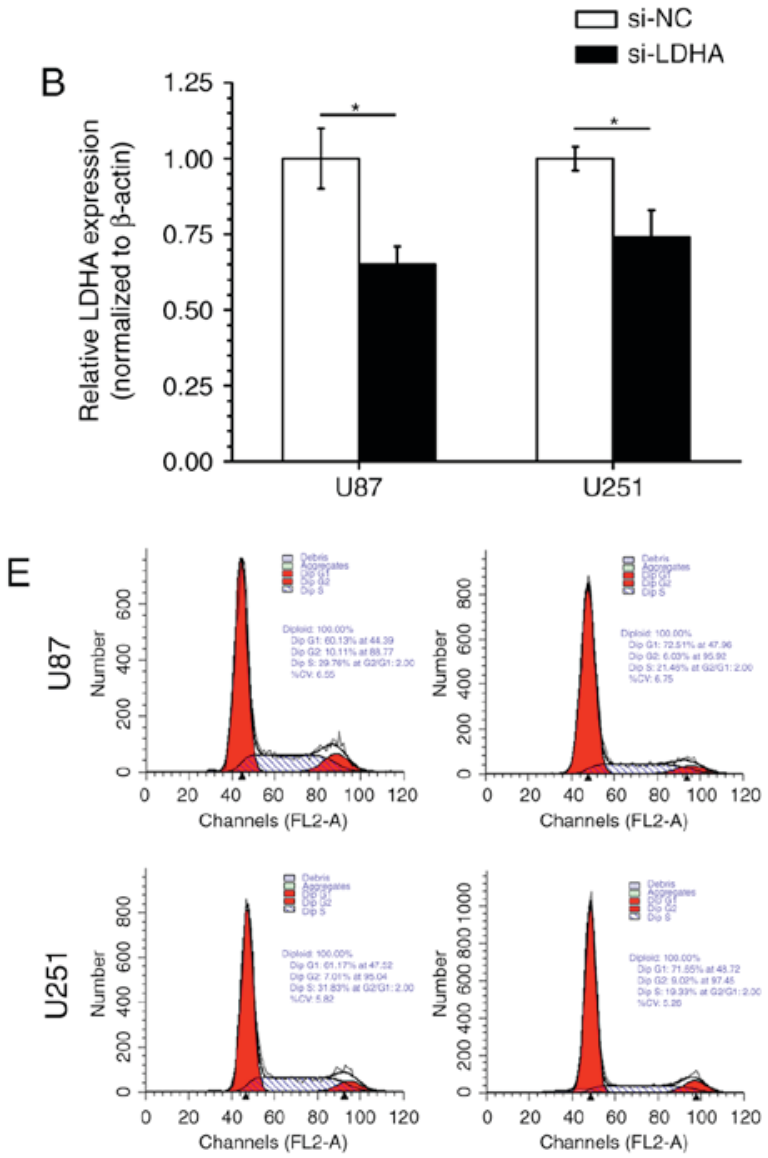

U87

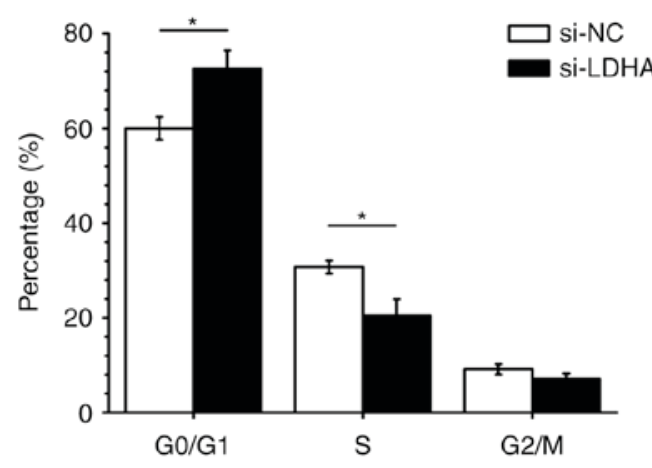

U251

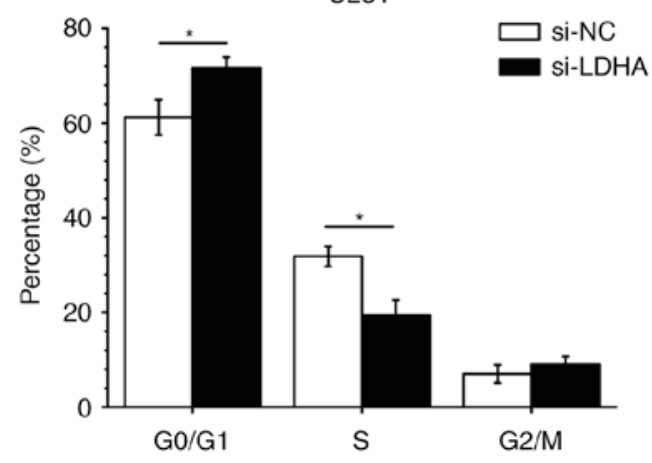

Figure 2. Effects of LDHA knockdown on U87 and U251 cell proliferation and apoptosis. (A and B) Western blotting (A) and qRT-PCR (B) analyses of LDHA following siRNA treatment $(72 \mathrm{~h})$ in U87 and U251 cells. (C) The viability of U87 and U251 cells was determined by MTT assays at $72 \mathrm{~h}$ after si-NC or si-LDHA transfection. (D) The percentage of apoptotic U87 and U251 cells was determined at $72 \mathrm{~h}$ after transfection using the Annexin V-FITC/PI Apoptosis Detection kit (BD Biosciences) and FACS measurement. Total percentages of Annexin V-positive cells are presented quantitatively. (E) Cell cycle progression was measured by flow cytometry. The percentages of cells in G0/G1, S, and G2/M phases are presented quantitatively. The data are presented as the mean \pm SD. ${ }^{*} \mathrm{P}<0.05,{ }^{* *} \mathrm{P}<0.01 . \mathrm{LDHA}$, lactate dehydrogenase A. 
A

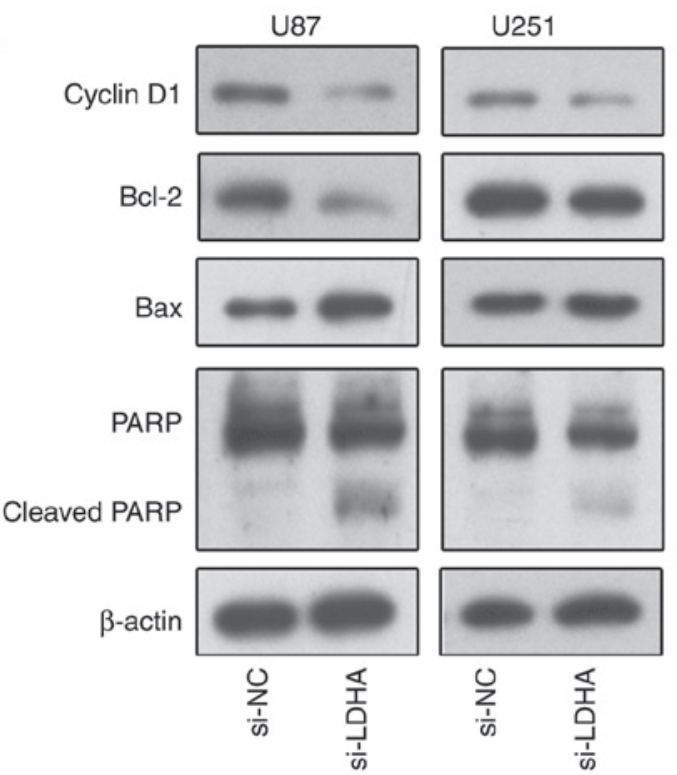

B

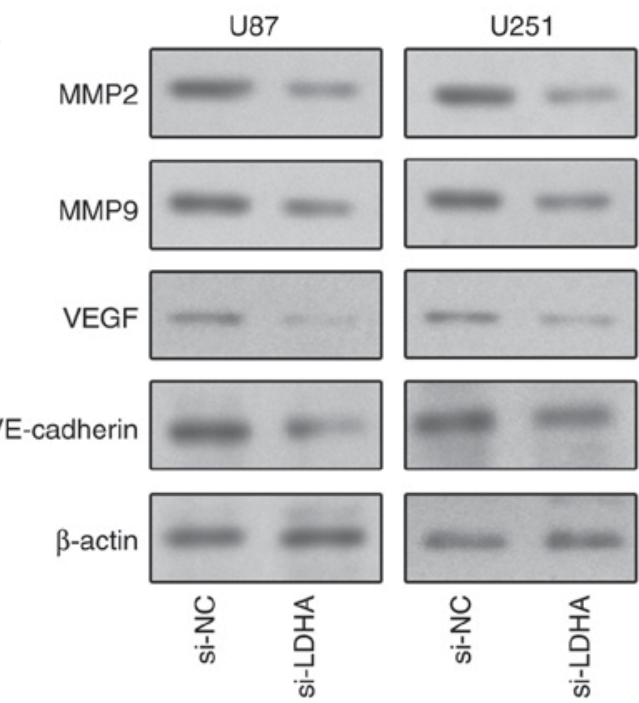

Figure 3. Effects of LDHA knockdown on viability-related and EMT-related proteins in U87 and U251 cells as determined by western blotting analyses (A) Cell cycle-related and apoptosis marker-related protein expression levels of cyclin D1, Bcl-2, Bax and cleaved PARP in U87 and U251 cells. (B) EMT-related protein expression levels of MMP-2, MMP-9, VE-Cadherin and VEGF in U87 and U251 cells. LDHA, lactate dehydrogenase A; EMT, epithelial-mesenchymal transition; MMP, matrix metalloproteinase.

Statistical analysis. Statistical analyses were performed using SPSS Statistics software (SPSS, Inc., Chicago, IL, USA). One-way ANOVA analysis was used to analyze the significance of the data between the two groups. Mann-Whitney U test was performed to analyze the relationship between LDHA and GBM malignancy. Differences with P-values $<0.05$ were considered to be statistically significant.

\section{Results}

The prognostic significance of LDHA expression levels in $G B M$. To investigate the association between LDHA expression levels and GBM malignancy, the expression of LDHA was examined by real-time PCR analysis. We categorized all glioma samples as grade II, grade III or grade IV according to the WHO classification guidelines. We found that the expression levels of LDHA in the high-grade samples (grade III and grade IV) were significantly higher than those in grade II tumors (Fig. 1). These results further confirm that LDHA might play an oncogenic role in the progression of GBM.

LDHA knockdown inhibits glioma cell growth and epithelial-mesenchymal transition (EMT). To further assess the potential oncogenic roles of LDHA in GBM, we used siRNA to knock down the expression of LDHA in two GBM cell lines: U87 and U251. We treated the cells with LDHA siRNA (si-LDHA) for $72 \mathrm{~h}$ and used scrambled siRNA as a negative control (si-NC); we then determined protein and mRNA expression levels via western blotting (Fig. 2A) and PCR (Fig. 2B). The data indicated that LDHA siRNA treatment successfully knocked down LDHA at both the protein and mRNA levels (Fig. 2A and B). Moreover, MTT assays showed that treatment of U87 and U251 cells with LDHA siRNA for $72 \mathrm{~h}$ led to an approximately 30 or $25 \%$ inhibition of cell growth compared to the results of si-NC

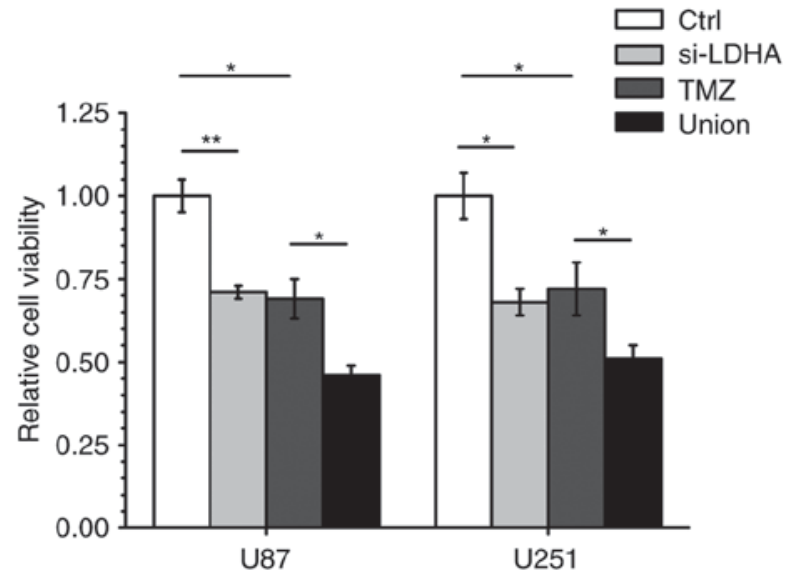

Figure 4. Effects of LDHA knockdown on the chemosensitivity of glioma cells to TMZ treatment. We transfected U87 and U251 cells with LDHA siRNA for $24 \mathrm{~h}$. Then, the cells were treated with TMZ at its $\mathrm{IC}_{50}(57.58 \mu \mathrm{M})$ for $72 \mathrm{~h}$, and cell viability was determined by MTT assays. The data are presented as the mean $\pm \mathrm{SD}$. ${ }^{*} \mathrm{P}<0.05,{ }^{* *} \mathrm{P}<0.01$. LDHA, lactate dehydrogenase A; TMZ, temozolomide.

treatment (Fig. 2C). Furthermore, we analysed the effects of LDHA knockdown on apoptosis and proliferation by using flow cytometry. As shown in Fig. 2D, according to the Annexin V-FITC/PI staining results, we found that treating U87 or U251 cells with si-LDHA led to a marked increase in the percentage of apoptotic cells compared to that of the si-NC group (Fig. 2D). In addition, cell cycle analyses showed that si-LDHA treatment significantly induced cell cycle arrest at the G0/G1 phase and inhibited S phase transition (Fig. 2E). Mechanistically, to further determine the impact of LDHA knockdown on cell growth-associated proteins, we treated the cells with si-LDHA for $48 \mathrm{~h}$ and analysed protein levels by western blotting (Fig. 3A). We found that LDHA siRNA treatment was related to decreased cyclin D1 expression and 
increased PARP cleavage; moreover, we found that the expression of Bcl-2 was decreased, whereas the expression of Bax was increased, indicating an enhancement in the apoptotic potential of LDHA-deficient U87 and U251 cells (Fig. 3A). These results indicated that LDHA knockdown inhibits the growth of glioma cells.

Furthermore, to determine the effects of LDHA on tumor progression-related proteins, we treated U87 and U251 cells with si-LDHA for $48 \mathrm{~h}$ and analysed protein levels by western blotting. As shown in Fig. 3B, we found that the levels of MMP-2, MMP-9, VE-Cadherin and VEGF were reduced in U87 and U251 cells after LDHA knockdown (Fig. 3B). These findings indicated that the knockdown of LDHA might inhibit EMT in GBM.

LDHA knockdown enhances the chemosensitivity of glioma cells to TMZ. Since it is known that TMZ chemotherapy is widely used for the treatment of advanced GBM, we determined whether knocking down LDHA would increase glioma cell sensitivity to TMZ. We transfected U87 and U251 cells with LDHA siRNA for $24 \mathrm{~h}$; then, we treated the cells with $\mathrm{TMZ}$ at its $\mathrm{IC}_{50}(57.58 \mu \mathrm{M})$ for $72 \mathrm{~h}$ and determined the cell viability by MTT assay. We found that under these experimental conditions, pre-treatment with LDHA siRNA followed by TMZ exposure (Union group) led to an approximately $40 \%$ inhibition of cell viability compared to the results of TMZ treatment alone (TMZ group) (Fig. 4). These results indicate that knocking down LDHA enhances the chemosensitivity of glioma cells to TMZ.

\section{Discussion}

In the present study, we demonstrated that there is a significant positive correlation between LDHA expression levels and GBM development. LDHA catalyzes the conversion of pyruvate to lactate and is considered to be a key checkpoint of anaerobic glycolysis (9). Increased levels of LDHA have been reported in several types of tumors $(11,13-15)$, and its expression levels have been correlated with clinical stages in breast cancer, renal cell carcinoma and prostate cancer; these reports further support that therapies targeting LDHA may provide useful strategies for controlling cancer progression. Moreover, previous reports using data from the Oncomine database showed that the mRNA levels of LDHA were up-regulated in GBM compared to those in matched normal tissues; these results prompted us to determine the correlation between LDHA expression levels and glioma malignancy (16). Consistent with previous studies, our results showed that there was a significant positive correlation between LDHA expression levels and GBM clinical stages. We also found that LDHA knockdown via siRNA inhibited cell proliferation and induced cell apoptosis. Upon examining the molecular mechanism, we found that LDHA siRNA treatment was related to decreased cyclin D1 expression, increased cleavage of PARP, and altered Bcl-2 and Bax expression, which contributes to growth arrest; in addition, LDHA knockdown leads to marked downregulation of the expression of matrix metalloproteinases (MMPs), VE-Cadherin and VEGF; this downregulation results in decreased cell migration and invasion in vitro. Thus, we provide evidence that LDHA may be involved in multiple mechanisms that modulate GBM cell viability, apoptosis and angiogenesis; however, the exact molecular mechanisms that modulate protease expression and angiogenesis need further examination.

Another interesting and important finding of this study was the effects of LDHA knockdown on chemosensitization to TMZ treatment. The current standard of prognosis, even after radiation therapy plus adjuvant $\mathrm{TMZ}$, remains poor, with a median survival of 14.6 months (3). It is important to explore novel strategies instead of studying conventional therapies to enhance the cytotoxicity of chemotherapeutic drugs. Our study showed that the combination of LDHA knockdown with TMZ treatment significantly reduced cell viability. Thus, targeting LDHA combined with TMZ treatment could be an effective therapeutic strategy for suppressing the growth of glioma. Although siRNA-based therapeutics are still in their initial stages of development, our findings are encouraging and suggest the potential of using LDHA as a diagnostic/prognostic marker and targeting LDHA with siRNA as a novel treatment for glioma in the future.

In conclusion, the current results strongly demonstrate that LDHA, an oncogene that contributes to the Warburg effect, has dramatic implications on glioma development via inhibiting cell proliferation, apoptosis, EMT and chemosensitivity to TMZ therapy. Our studies further confirm that the expression levels of LDHA should be of high clinical relevance in the treatment of GBM; these findings may open new avenues for developing novel treatments.

\section{Acknowledgements}

The present study was funded by the Project of Hebei Province Science and Technology Program (162777254); the Medical Science Research Key Program of Hebei Province (20160379); the Clinical Talent Cultivation and Basic Research Project of Hebei Province (361007); the Foundation for High-level Talents in Higher Education of Hebei Province (CY201601); and the 2017 Science and Technology Research and Development Guidance Project of Baoding (17ZF003).

\section{References}

1. Ellis HP, Greenslade M, Powell B, Spiteri I, Sottoriva A and Kurian KM: Current challenges in glioblastoma: Intratumour heterogeneity, residual disease, and models to predict disease recurrence. Front Oncol 5: 251, 2015.

2. Jordan JT, Gerstner ER, Batchelor TT, Cahill DP and Plotkin SR: Glioblastoma care in the elderly. Cancer 122: 189-197, 2016.

3. Khosla D: Concurrent therapy to enhance radiotherapeutic outcomes in glioblastoma. Ann Transl Med 4: 54, 2016

4. Razungles J, Cavaillès V, Jalaguier S and Teyssier C: The warburg effect: From theory to therapeutic applications in cancer. Med Sci (Paris) 29: 1026-1033, 2013 (In French).

5. Courtnay R, Ngo DC, Malik N, Ververis K, Tortorella SM and Karagiannis TC: Cancer metabolism and the warburg effect: The role of HIF-1 and PI3K. Mol Biol Rep 42: 841-851, 2015.

6. Upadhyay M, Samal J, Kandpal M, Singh OV and Vivekanandan P: The warburg effect: Insights from the past decade. Pharmacol Ther 137: 318-330, 2013.

7. Teng Y, Zhang Y, Qu K, Yang X, Fu J, Chen W and Li X: MicroRNA-29b (mir-29b) regulates the warburg effect in ovarian cancer by targeting AKT2 and AKT3. Oncotarget 6: 40799-40814, 2015.

8. Valvona CJ, Fillmore HL, Nunn PB and Pilkington GJ: The regulation and function of lactate dehydrogenase $A$ : Therapeutic potential in brain tumor. Brain Pathol 26: 3-17, 2016. 
9. Miao P, Sheng S, Sun X, Liu J and Huang G: Lactate dehydrogenase A in cancer: A promising target for diagnosis and therapy. IUBMB Life 65: 904-910, 2013.

10. Wang ZY, Loo TY, Shen JG, Wang N, Wang DM, Yang DP, Mo SL, Guan XY and Chen JP: LDH-A silencing suppresses breast cancer tumorigenicity through induction of oxidative stress mediated mitochondrial pathway apoptosis. Breast Cancer Res Treat 131: 791-800, 2012.

11. Yao F, Zhao T, Zhong C, Zhu J and Zhao H: LDHA is necessary for the tumorigenicity of esophageal squamous cell carcinoma Tumour Biol 34: 25-31, 2013.

12. Sheng SL, Liu JJ, Dai YH, Sun XG, Xiong XP and Huang G: Knockdown of lactate dehydrogenase A suppresses tumor growth and metastasis of human hepatocellular carcinoma. FEBS J 279: 3898-3910, 2012.
13. Rong Y, Wu W, Ni X, Kuang T, Jin D, Wang D and Lou W: Lactate dehydrogenase A is overexpressed in pancreatic cancer and promotes the growth of pancreatic cancer cells. Tumour Biol 34: 1523-1530, 2013.

14. Wang X, Xu L, Wu Q, Liu M, Tang F, Cai Y, Fan W, Huang H and $\mathrm{Gu} \mathrm{X}$ : Inhibition of LDHA deliver potential anticancer performance in renal cell carcinoma. Urol Int 99: 237-244, 2017.

15. Xian ZY, Liu JM, Chen QK, Chen HZ, Ye CJ, Xue J, Yang HQ Li JL, Liu XF and Kuang SJ: Inhibition of LDHA suppresses tumor progression in prostate cancer. Tumour Biol 36: 8093-8100, 2015.

16. Li J, Zhu S, Tong J, Hao H, Yang J, Liu Z and Wang Y: Suppression of lactate dehydrogenase A compromises tumor progression by downregulation of the warburg effect in glioblastoma. Neuroreport 27: 110-115, 2016. 